\title{
Bayesian validation of a serum and milk ELISA for antibodies against Mycobacterium avium subspecies paratuberculosis in Greek dairy goats across lactation
}

\author{
E. Angelidou, ${ }^{1}$ P. Kostoulas, and L. Leontides \\ Laboratory of Epidemiology, Biostatistics and Animal Health Economics, Faculty of Veterinary Medicine, University of Thessaly, \\ Trikalon 224, GR-43100, Karditsa, Greece
}

\begin{abstract}
We validated a commercial (Idexx Pourquier, Montpellier, France) serum and milk indirect ELISA that detects antibodies against Mycobacterium avium ssp. paratuberculosis (MAP) in Greek dairy goats. Each goat was sampled 4 times, starting from kidding and covering early, mid, and late lactation. A total of 1,268 paired milk (or colostrum) and serum samples were collected during the 7-mo lactation period. Bayesian latent class models, which allow for the continuous interpretation of test results, were used to derive the distribution of the serum and milk ELISA response for healthy and MAP-infected individuals at each lactation stage. Both serum and milk ELISA, in all lactation stages, had average and similar overall discriminatory ability as measured by the area under the curve (AUC). For each test, the smallest overlap between the distribution of the healthy and MAP-infected does was in late lactation. At this stage, the AUC was $0.89(95 \%$ credible interval: $0.70 ; 0.98)$ and $0.92(0.74 ; 0.99)$ for the milk and serum ELISA, respectively. Both tests had comparable sensitivities and specificities at the recommended cutoffs across lactation. Lowering the cutoffs led to an increase in sensitivity without serious loss in specificity. In conclusion, the milk ELISA was as accurate as the serum ELISA. Therefore, it could serve as the diagnostic tool of choice, especially during the implementation of MAP control programs that require frequent testing, because milk sampling is a noninvasive, rapid, and easy process.
\end{abstract}

Key words: Bayesian latent class model, paratuberculosis, serum and milk ELISA, dairy goat

\section{INTRODUCTION}

Paratuberculosis, which is caused by Mycobacterium avium ssp. paratuberculosis (MAP), is a chronic

Received July 2, 2013.

Accepted November 7, 2013.

${ }^{1}$ Corresponding author: eaggel@vetuth.gr intestinal infection in cattle, sheep, goats, and other ruminants. The disease decreases productivity, leads to suboptimal productive life, and, consequently, causes substantial economic losses to the farming industry (Clarke, 1997). The Greek national herd comprises approximately 5 million goats, which are reared primarily for milk production (Zygogiannis and Katsaounis, 1992). The majority of goat flocks are endemically infected with MAP (Chiodini et al., 1984). Goats can become persistent fecal shedders about 1 yr postinfection without any clinical signs of paratuberculosis (Storset et al., 2001) apparent during a long latent subclinical phase. In goats, early clinical signs of the disease include progressive wasting and decrease in milk production, which are followed by manifestations of advanced clinical disease: flaky skin, poor hair coat, progressive emaciation, dehydration, anemia with submandibular edema, depression, and diarrhea (Stehman, 1996). Commonly used diagnostic tests, such as ELISA and fecal culture, have low sensitivities (Se) for identifying infected individuals during the early latent infection stage (Bakker et al., 2000).

An impediment to the surveillance and control of paratuberculosis is the cost of testing, particularly for small ruminant industries, because of the low economic value of each animal (Salgado et al., 2007). Specifically, diagnosis of paratuberculosis by fecal culture is slow, laborious, and expensive. In contrast, serum ELISA is quick and automated but sample collection may be laborious and increase the cost of surveillance. Compared with serum ELISA, milk ELISA has the advantage of easy sample collection. Its validation could make paratuberculosis testing more affordable and more widely applicable as a useful tool for the management of this disease by dairy goat farmers.

In the absence of a perfect reference test to ascertain MAP infection status, many researchers have acknowledged the need for latent class methods (Branscum et al., 2005; Kostoulas et al., 2006; Wang et al., 2007). These methods account for all latent infection stages to obtain estimates of the validity of diagnostic tests. Recently, Bayesian latent class models have been pro- 
posed that do not require dichotomization of the test outcomes and apply to the actual continuous or ordinal test results (Choi et al., 2006; Jafarzadeh et al., 2010; Wang et al., 2011). The advantage of this approach is that the actual distributions of the healthy and infected populations can be derived. Thus, the continuous interpretation of test results is feasible, avoiding simple definitions of MAP infection, which can be difficult and even misleading due to the chronicity of the infection (Toft et al., 2005). For example, dichotomization of ELISA results leads to loss of valuable information conveyed in the test by disregarding the fact that not all positive results are equal.

In dairy cattle, milk ELISA has been evaluated across lactation (Nielsen et al., 2002a), compared with serum ELISA (Kennedy and Benedictus, 2001; Hendrick et al., 2005; Lombard et al., 2006) with models that allow continuous interpretation of the results (Kostoulas et al., 2013). However, we cannot extrapolate results from dairy cattle to dairy goats. Researchers have indicated the need for evaluations of MAP diagnostics that are specific to species (Kostoulas et al., 2006), strain (Florou et al., 2009), target condition (Nielsen et al., 2007), and lactation stage (Nielsen et al., 2002a). In dairy goats, few studies have evaluated the milk ELISA (Salgado et al., 2005, 2007; Kumar et al., 2008) and none have done so across lactation, with the use of latent class models and continuous interpretation of results. Therefore, the objective of this study was to assess and compare the overall diagnostic validity of a commercial ELISA kit between milk and serum samples at different lactation stages in Greek dairy goats. The latent class analyses were done in a Bayesian framework.

\section{MATERIALS AND METHODS}

\section{Study Population and Sampling Scheme}

A flock of 300 dairy goats was selected for the study. The flock had a history of clinical paratuberculosis and was unvaccinated against MAP. The does were of the domestic breed or their crosses with the Alpine breed. The age of the does ranged from 1 to 8 yr old (median $4 \mathrm{yr}$ ). The animals were kept under semi-intensive management for milk production, which was the primary breeding goal. The farmers selected replacements from among the daughters of high-yielding does. The males brought into the flocks originated from highyielding animals from other flocks. The animals grazed on pasture most of the year and were additionally fed concentrates. They spent most of the day outside and were moved into the shed during the night. They were mated to bucks, in an unsupervised manner, in June through September and delivered during December and
March of the following year. The kids were weaned 45 to $60 \mathrm{~d}$ after birth; subsequently, the does were milked by hand. Milking was ceased abruptly when the stockman felt that the milk yield was so reduced that it did not compensate for the cost of the milking routine and extra feeding.

The does were followed from December 2008 to March 2010. During this period, we collected 1,268 paired milk (or colostrum) and blood samples. The lactating does within the flock were separated into 2 groups according to kidding month. We sampled the first group of goats that kidded in December 2008 and followed them during the 7-mo lactation period until November 2009, covering early, mid, and late lactation. We followed the second group, which kidded during March 2009, from May 2009 until March 2010.

\section{Diagnostic Tests}

Collected milk and colostrum samples were centrifuged, skimmed $\left(-8^{\circ} \mathrm{C}, 1,600 \times g\right.$ for $\left.20 \mathrm{~min}\right)$, and stored at $-21^{\circ} \mathrm{C}$, until testing. Sera were tested using a commercial indirect ELISA kit (Idexx Pourquier, Montpellier, France) according to the manufacturer's protocol. Skimmed colostrum and milk samples were tested by the same ELISA using the manufacturer's protocol for bovine milk (Salgado et al. 2007). The paired sera and milk samples were tested simultaneously to avoid in-plate and in-day variability (Nielsen, 2002). The recorded optical densities (OD) were transformed to the sample-to-positive $(\mathbf{S} / \mathbf{P})$ ratio, which were kept on a continuous scale, instead of using several cutoffs to dichotomize the results, for further analysis (Toft et al., 2005).

\section{Statistical Analyses}

We implemented a Bayesian mixture modeling approach to predict the distribution of the serum and milk ELISA response by infection status separately for each lactation stage.

Definition of Infection Status. Bayesian mixture models for diagnostic test evaluation create their own probabilistic definition of infection, which implicitly assumes a biological definition that has to be explicitly described. Essentially, this is determined by the target condition that the analytes and biomarkers of the test under consideration measure (Gardner et al., 2011). In our case, to describe MAP infection in biological terms, we used the approach described by Nielsen et al. (2002b) and Kostoulas et al. (2006). Hence, by "MAP infection" we mean that goats carry MAP intracellularly; substantial replication need not take place because the infection can be latent. Entrance and persistence 
of MAP have lasted long enough to give a detectable humoral immune response at any time during their life; we assumed that once an animal has an established infection, the infection persists for life.

Bayesian Mixture Model. The proposed model determines the distribution of the continuous serum and milk ELISA response by infection status and lactation stage, adjusting for the likely correlation of the OD measurements within animal and lactation stage. For each infection status, we assumed that either the original continuous test responses were normally distributed or could be transformed to normality using appropriate methods such as the log-transformation (Toft et al., 2005; Nielsen et al., 2007). Let $Y_{i j}$ denote the log-transformed ELISA response of the $i$ th doe at the $j$ th lactation stage, with $j=1, \ldots, 4$ corresponding to kidding, early, mid, and late lactation stages for the serum ELISA and $j=5, \ldots, 8$ for the same stages for milk ELISA. Also, let $D_{i j}$ be the latent data that represents the unknown true disease status of each doe at each lactation stage, with $D_{i j}=0$ for the non-MAPinfected and $D_{i j}=1$ for the MAP-infected individuals. The $Y_{i j}$ follow a mixture multivariate normal distribution, with 2 mixture components:

$$
\begin{gathered}
\left(Y_{i j} \mid D_{i j}\right) \sim \varphi\left(\cdot \mid \mu_{j 1}, \Sigma_{j j 1}\right)^{D_{i j}} \varphi\left(\cdot \mid \mu_{j 0}, \Sigma_{j j 0}\right)^{1-D_{i j}}, \\
D_{i j} \sim \operatorname{Bernoulli}\left(\pi_{j}\right), \\
\boldsymbol{\Sigma}_{\mathbf{j j 0}}=\left(\begin{array}{ccc}
\sigma_{110}^{2} & \cdots & \sigma_{1 j 0} \\
\vdots & \ddots & \vdots \\
\sigma_{j 10} & \cdots & \sigma_{j j 0}^{2}
\end{array}\right) \\
\boldsymbol{\Sigma}_{\mathbf{j j 1}}=\left(\begin{array}{ccc}
\sigma_{111}^{2} & \cdots & \sigma_{1 j 1} \\
\vdots & \ddots & \vdots \\
\sigma_{j 11} & \cdots & \sigma_{j j 1}^{2}
\end{array}\right)
\end{gathered}
$$

The $D_{i j}$ follow the Bernoulli distribution, where $\pi_{j}$ is the prevalence of the infection at each lactation stage, $\varphi$ is the multivariate normal probability density function with parameters: $\boldsymbol{\mu}_{\mathbf{j} 0}\left(\boldsymbol{\mu}_{\mathbf{j} 1}\right)$ the mean vector and $\boldsymbol{\Sigma}_{\mathbf{j} j 0}$ $\left(\boldsymbol{\Sigma}_{\mathbf{j j} 1}\right)$ the variance co-variance matrix for the distribution of the healthy (diseased) animals. Because $j=1$, ..., 4 corresponds to the serum ELISA measurements and $j=5, \ldots, 8$ to milk ELISA measurements on the same individuals, $\pi_{j}=\pi_{j+4}$ for $j=1, \ldots, 4$. Given the distribution of the MAP-infected and the non-MAPinfected individuals by lactation stage $j$, the $S e_{j}$ and the $S p_{j}$ for any cutoff value $c \in(-\infty,+\infty)$ are defined as

$$
\begin{gathered}
S e_{j}(c)=1-\Phi\left(\frac{c-\mu_{j 1}}{\sqrt{\sigma_{j j 2}^{2}}}\right), \\
S p_{j}(c)=\Phi\left(\frac{c-\mu_{j 0}}{\sqrt{\sigma_{j j 0}^{2}}}\right),
\end{gathered}
$$

where $\Phi$ is the cumulative distribution function. Subsequently, the receiver operator characteristic (ROC) curves can be constructed by plotting the pairs of the estimated $\left(1-S p_{j}, S e_{j}\right)$. The AUC for the serum and milk ELISA at each lactation stage is

$$
A U C_{j}=\Phi\left(\frac{\mu_{j 1}-\mu_{j 0}}{\sqrt{\sigma_{j j 1}^{2}+\sigma_{j j 0}^{2}}}\right) .
$$

For either serum or milk ELISA, we selected as a potential optimum cutoff an $\mathrm{S} / \mathrm{P}$ percentage that optimizes prevalence-independent summary measures of $S e$ and $S p$ such as the Youden index $J=\max \left[S p_{j}(c)+S e_{j}(c)-\right.$ 1]. This occurs where the ROC curve gets closest to the top left corner of the graph (Fluss et al., 2005).

Finally, the correlation $\rho_{k D}$ between the serum and milk ELISA for the non-MAP-infected $(D=0)$ and the MAP-infected $(D=1)$ individuals at the $k$ th lactation stage can be estimated by the elements of the variance co-variance matrix: $\rho_{k D}=\sigma_{k l D}^{2} /\left(\sigma_{k k D} \sigma_{l l D}\right)$, with $k=1$, $\ldots, 4$ and $l=k+4$.

Constant Prevalence Across Lactation Stages. Paratuberculosis develops slowly and the prevalence of the disease is expected to remain unchanged across one lactation period. Thus, we also consider a slight modification of our initial model to allow for a constant prevalence across the whole observation period:

$$
\begin{gathered}
D_{i} \sim \operatorname{Bernoulli}(\pi), \\
\left(Y_{i j} \mid D_{i j}\right) \sim \varphi\left(\cdot \mid \mu_{j 1}, \Sigma_{j j 1}\right)^{D_{i}} \varphi\left(\cdot \mid \mu_{j 0}, \Sigma_{j j 0}\right)^{1-D_{i}},
\end{gathered}
$$

Prior Selection. We selected noninformative priors for the parameters $\pi, \pi_{\mathrm{k}}, \boldsymbol{\mu}_{\mathrm{jD}}$, and $\boldsymbol{\Sigma}_{\mathrm{jj \textrm {D }}}$, that follow the Beta $(B e)$, Normal $(N)$ and Wishart distributions, respectively:

$$
\begin{gathered}
\pi \sim B e(1,1), \\
\pi_{k} \sim B e(1,1), \\
\boldsymbol{\mu}_{\mathbf{j D}} \sim N(0,100),
\end{gathered}
$$




$$
\boldsymbol{\Sigma}_{\mathrm{jjD}} \sim \operatorname{Wishart}(8, \boldsymbol{\Gamma}),
$$

where $\boldsymbol{\Gamma}$ is an $8 \times 8$ matrix and 8 represents the degrees of freedom. To represent vague prior knowledge, we chose the degrees of freedom to be as small as possible, 8 being the rank of $\boldsymbol{\Sigma}_{\mathrm{jjD}}$.

Sensitivity Analysis. We also considered less diffuse prior values, which is recommended when low information priors are used (Ntzoufras, 2009). Two alternative sets of priors were used in the sensitivity analysis. The first set was the same as for the primary analysis but with less diffuse priors specified on the mean of the healthy individuals and on the prevalence of infection: $\boldsymbol{\mu}_{\mathbf{j} 0} \sim N(0,10), \pi \sim \operatorname{Be}(2,2)$, and $\pi_{k} \sim$ $\operatorname{Be}(2,2)$. The second set was more informative on the same priors: $\boldsymbol{\mu}_{\mathrm{j} 0} \sim N(0.01,0.07), \pi \sim \operatorname{Be}(15,2.6)$, and $\pi_{k} \sim \operatorname{Be}(15,2.6)$.

Assessment of Convergence. Convergence diagnostics for Markov chain Monte Carlo (MCMC) sampling are not foolproof. Therefore, a combination of diagnostics plus visual inspection of the trace plots and summary statistics is recommended (Best et al., 1995). To assess the convergence of the MCMC, we checked the autocorrelations and the trace plots. We also checked the parameter summary statistics of 90,000 iterations after a burn-in phase of 10,000 iterations. This was adequate because the Raftery and Lewis (1992) method suggested that analytical summaries of 45,000 iterations after a burn-in of 15 iterations were needed. To assess the effect of prior value selection on the conclusions, we obtained the posterior medians and credible intervals (CrI) of the AUC (Choi et al., 2006).

\section{Statistical Software}

The model was run in the freeware program WinBugs (Spiegelhalter et al., 1996). The graphs in the manuscript were produced in the statistical package $\mathrm{R}$ (http://www.r-project.org/).

\section{RESULTS}

Figure 1 shows the distributions of the MAP-infected and healthy does for the serum and milk ELISA for each lactation stage. The estimated $\boldsymbol{\mu}_{\mathrm{j} 0}$ and $\boldsymbol{\mu}_{\mathbf{j} 1}$ and the corresponding $95 \% \mathrm{CrI}$ for each of these distributions are presented in Table 1. Originally, means and CrI were obtained for the log-transformed values that were then back-transformed to the actual $\mathrm{S} / \mathrm{P}$ scale. The mean $\mathrm{S} / \mathrm{P}$ values of both serum and milk ELISA did not differ among lactation stages.

The estimated AUC and CrI by lactation stage are given in Table 2. Both tests in all lactation stages had average (0.7-0.9) overall discriminating ability as measured by the AUC. Both tests had comparable AUC across the different lactation stages. Further, for both tests, we observed no significant differences between the different lactation stages with the exception of the estimated AUC for the milk ELISA during kidding that had a lowered mean value of 63 (95\% CrI: 39; 82). For both tests, the highest power to discriminate healthy from infected does was in late lactation.

The ROC curves for both tests by lactation stage are given in Figure 2. Evidently, despite the comparable overall discriminating ability, the tests had different diagnostic accuracy at selected cutoffs. The Se and Sp at the recommended cutoffs $(\mathrm{S} / \mathrm{P}=45 \%$ in serum and $20 \%$ in milk) and at the $50 \%$ reduced cutoffs (Kostoulas et al., 2006) are given in Table 2. When the cutoff values were reduced, Se were increased without serious loss of Sp. Specifically, the optimum cutoffs that simultaneously maximized Se and Sp were $0.45,0.45$, 0.46 , and 0.47 for serum ELISA and 0.44, 0.43, 0.44, and 0.46 for milk ELISA at kidding, early, mid, and late lactation stages, respectively.

Estimates under the model assuming distinct prevalence for each lactation stage and that with constant prevalence were comparable (Tables 1 and 2), indicating that the non-lactation-stage-specific prevalence was similar.

\section{DISCUSSION}

We assessed the overall discriminatory power of serum and milk ELISA for the diagnosis of MAP infection in dairy goats at different lactation stages (kidding, early, mid, and late lactation). The estimated AUC, which serves as a global average statistic of the diagnostic validity of a test, indicated that both the serum and milk ELISA, at each lactation stage, are moderately accurate tests, because they fall within the 0.7 to 0.9 interval (Greiner et al., 2000). The AUC estimates were in accordance with a previous AUC that measured the diagnostic accuracy of a serum ELISA in Greek goats (Kostoulas et al., 2006). In that study, they also used latent class models to adjust for all latent infection stages. The latent class models do not lead to overestimates of the diagnostic accuracy of tests, which can occur when the accuracy estimates are based on confirmation procedures that are used as gold standards but do not include all latent cases of infection.

The overall discriminatory power of the serum and milk ELISA tests was moderate because of the existence of latently infected animals that were at an early stage of infection. In the early stages of MAP infection, undetectable levels of antibodies are produced. Interferon- $\gamma$ and tumor necrosis factor- $\alpha$ (TFN- $\alpha$ ), 
Table 1. The estimated medians of the mean values of the distributions of the healthy and the diseased ELISA responses for each stage of lactation in serum and milk ELISA of the log normal sample-to-positive ratios $(\mathrm{S} / \mathrm{P}),{ }^{1}$ which was then back transformed

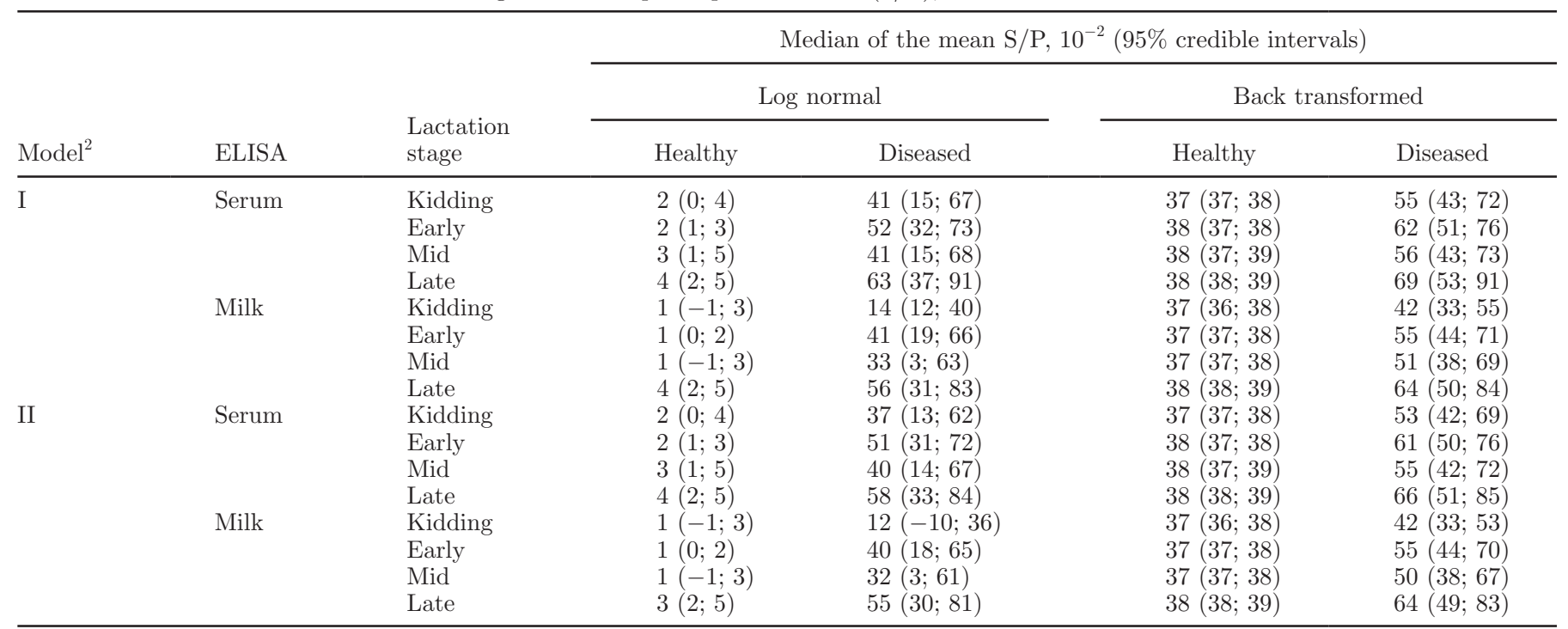

${ }^{1} Y_{i j}=\log _{e}[(\mathrm{~S} / \mathrm{P})+1]$.

${ }^{2}$ In model I (II), we assumed different (constant) prevalence for each lactation stage.
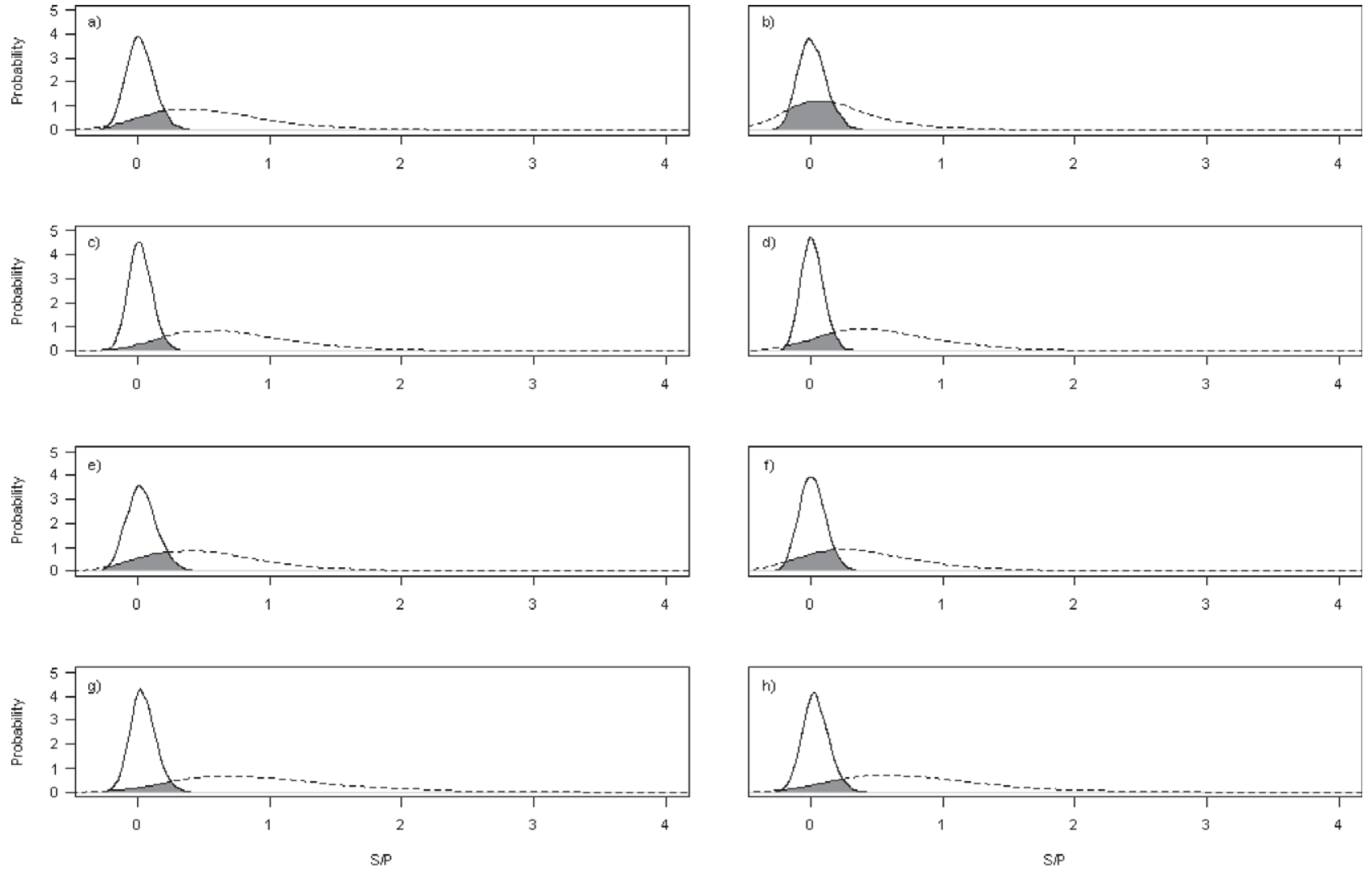

Figure 1. The predicted distributions of the sample-to-positive ratios $(\mathrm{S} / \mathrm{P})$ of the healthy and the infected population in serum (a, c, e, g) and milk (b, d, f, h) ELISA at $(\mathrm{a}, \mathrm{b})$ kidding, $(\mathrm{c}, \mathrm{d})$ early, $(\mathrm{e}, \mathrm{f})$ mid, and $(\mathrm{g}, \mathrm{h})$ late stage of lactation. Initial predictions were based on the variable $Y_{i j}=\log _{e}[(\mathrm{~S} / \mathrm{P})+1]$, which was then back-transformed to the original S/P percentage. The gray area is the overlap between the healthy and infected populations. The better the discrimination ability of the test, the smaller the overlap. 
Table 2. The medians (95\% credible interval in parentheses) of the area under the curve (AUC), and sensitivity (Se) and specificity (Sp) for the recommended and $50 \%$ reduced cutoffs at different stages of lactation in serum and milk ELISA

\begin{tabular}{|c|c|c|c|c|c|c|}
\hline \multirow[b]{2}{*}{ Item } & \multirow[b]{2}{*}{ Model $^{1}$} & \multirow[b]{2}{*}{ ELISA } & \multicolumn{4}{|c|}{ Lactation stage } \\
\hline & & & Kidding & Early & Mid & Late \\
\hline \multirow{3}{*}{ AUC, $10^{-2}$} & & Milk & $64(38 ; 84)$ & $87(66 ; 97)$ & $78(52 ; 93)$ & $89(70 ; 98)$ \\
\hline & II & Serum & $81(60 ; 94)$ & $93(76 ; 99)$ & $82(59 ; 95)$ & $89(71 ; 98)$ \\
\hline & & Milk & $63(39 ; 82)$ & $87(66 ; 97)$ & $77(51 ; 93)$ & $88(69 ; 97)$ \\
\hline \multirow[t]{2}{*}{$\mathrm{Se}^{2} \%$} & I & Serum & $54(30 ; 77)$ & $67(44 ; 86)$ & $54(30 ; 77)$ & $74(50 ; 92)$ \\
\hline & & Milk & $43(21 ; 68)$ & $74(50 ; 92)$ & $63(37 ; 85)$ & $81(59 ; 94)$ \\
\hline \multirow[t]{4}{*}{$\mathrm{Se}^{3} \%$} & I & Serum & $70(45 ; 89)$ & $83(62 ; 95)$ & $70(45 ; 89)$ & $85(64 ; 97)$ \\
\hline & & Milk & $55(30 ; 79)$ & $82(59 ; 96)$ & $72(45 ; 91)$ & $87(66 ; 97)$ \\
\hline & II & Serum & $67(43 ; 86)$ & $83(61 ; 95)$ & $70(45 ; 89)$ & $81(60 ; 94)$ \\
\hline & & Milk & $53(29 ; 76)$ & $82(58 ; 96)$ & $71(44 ; 90)$ & $86(66 ; 97)$ \\
\hline \multirow[t]{2}{*}{$\mathrm{Sp},{ }^{2} \%$} & I & Serum & $100(100 ; 100)$ & $100(100 ; 100)$ & $100(99 ; 100)$ & $100(100 ; 100)$ \\
\hline & & Milk & $95(91 ; 97)$ & $98(96 ; 99)$ & $95(91 ; 98)$ & $93(89 ; 96)$ \\
\hline \multirow{2}{*}{$\mathrm{Sp},{ }^{3} \%$} & II & Serum & $95(91 ; 97)$ & $98(96 ; 99)$ & $95(91 ; 98)$ & $93(89 ; 96)$ \\
\hline & & Milk & $79(72 ; 84)$ & $84(79 ; 88)$ & $79(72 ; 85)$ & $73(66 ; 78)$ \\
\hline
\end{tabular}

${ }^{1}$ In model I (II), we assumed different (constant) prevalence for each lactation stage.

${ }^{2}$ The Se and Sp estimated at the recommended cutoffs ( $\mathrm{S} / \mathrm{P}=45 \%$ for serum and $\mathrm{S} / \mathrm{P}=20 \%$ for milk). $\mathrm{S} / \mathrm{P}=$ sample-to-positive ratio.

${ }^{3}$ The Se and $\mathrm{Sp}$ at the $50 \%$ reduced cutoffs $(\mathrm{S} / \mathrm{P}=22.5 \%$ for serum and $\mathrm{S} / \mathrm{P}=10 \%$ for milk).

which activate macrophages and achieve control of the infection, usually precede a humoral response in goats (Storset et al., 2001; Lybeck et al., 2011) and cattle, although low levels of detectable antibodies could occur at this stage. Clearance may or may not occur because some macrophages remain inactive and infected. These macrophages decay sporadically within granulomas, which accounts for transient bacterial shedding. As MAP infection progresses, the cell-mediated immune reactions are no longer capable of controlling MAP proliferation, and the shift to a humoral immune response and production of detectable levels of antibodies occurs (Stabel, 2000; Coussens, 2001).

The estimated AUC were comparable between serum and milk ELISA across all lactation stages. Therefore, the milk ELISA may be used instead of the serum ELISA for the diagnosis of MAP infection in dairy goats. Both tests have similar discriminatory power but the former has the advantage that milk samples are easily collected, in a noninvasive way, which offers the farmer the opportunity to screen for MAP with lower labor and sampling costs.

The estimated means of the $\mathrm{S} / \mathrm{P}$ ratio in milk were higher in late lactation than at kidding for the infected population (Table1). Nielsen et al. (2002a) suggested a similar trend in the milk of dairy cattle. Those authors also found high antibody levels at the beginning of lactation, which was not observed in this study. Differences in the milking frequency, milk volume, and husbandry between dairy cattle and goats could partly explain this. An inverse relationship exists between milk volume and IgG concentration in dairy cattle (Pritchett et al., 1991), whereas milk IgG concentration is negatively correlated with milking frequency in goats (HernándezCastellano et al., 2011). Furthermore, the IgG levels in goat milk depend on the milking frequency and stage of lactation. In Greek dairy goat flocks, does are housed indoors with their newborn kids for the first $5 \mathrm{~d}$ after kidding. We collected colostrum samples $10 \mathrm{~h}$ after kidding at a time at which the kids had increased suckling frequency. A similar trend was observed in Majorera goats where the colostrum IgG concentration declined rapidly in the first $10 \mathrm{~h}$ after kidding (Moreno-Indias et al., 2012). Greek dairy goats are milked once daily in late lactation, leading to a high antibody concentration in milk.

We estimated the optimum cutoffs that simultaneously maximize the Se and Sp of the ELISA for sera and milk testing at each lactation stage. The optimum cutoffs were similar to those recommended by the manufacturer for the serum ELISA. However, the recommended cutoff for the milk ELISA was lower than the optimum. The manufacturer proposes one cutoff for the blood and serum ELISA, which is not species specific. However, differences may exist in the distribution of MAP strains, immune response, ability to contain 
a)

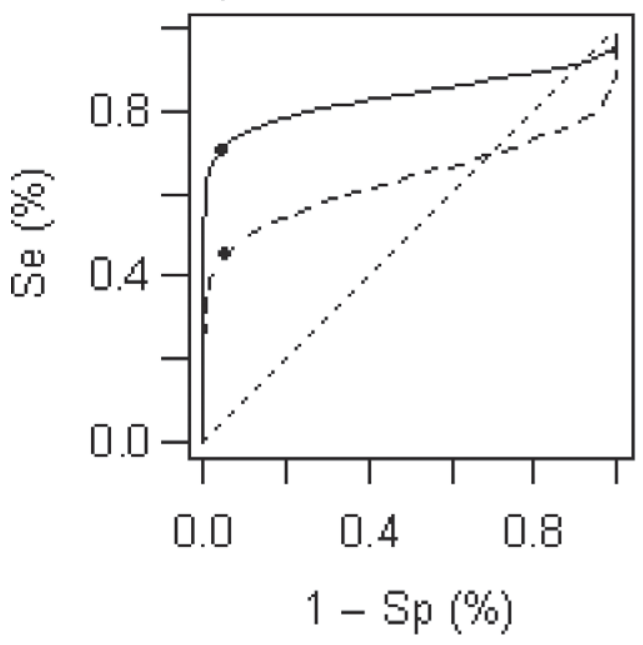

c)

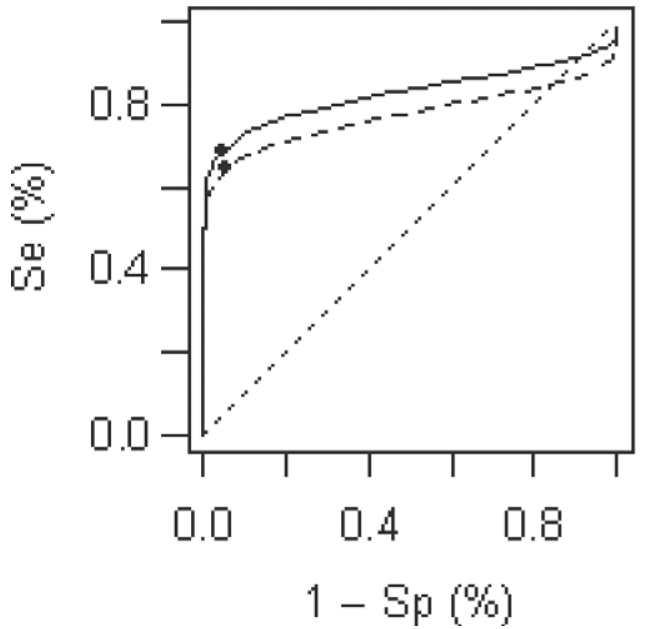

b)

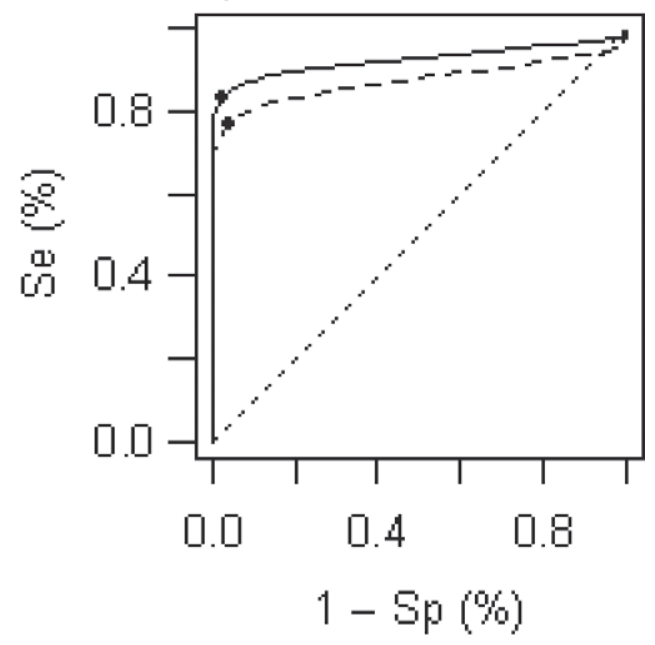

d)

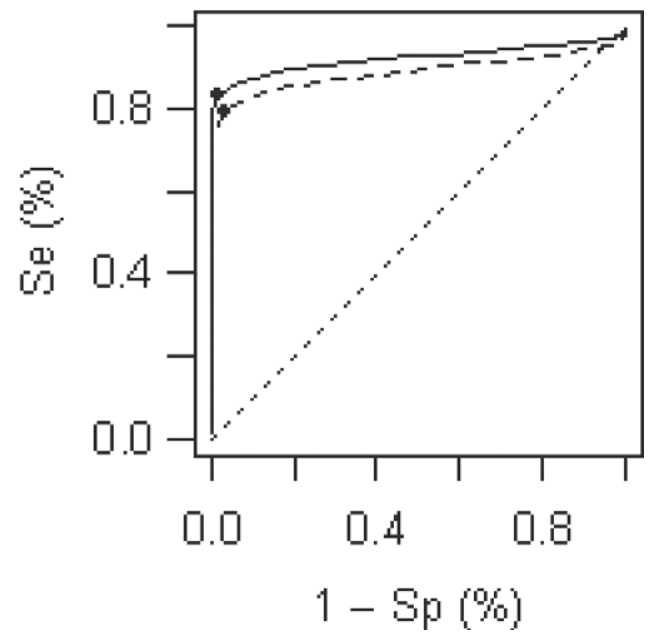

Figure 2. Receiver operating curves in serum (solid line) and milk (long-dash) ELISA at (a) kidding, (b) early, (c) mid, and (d) late stage of lactation. For comparison, the short-dash line depicts the curve of a noninformative test, with the area under the curve equal to 0.5. Marked points $(\bullet)$ correspond to the values closest to the upper left corner of the unit square. Se = sensitivity; Sp $=$ specificity.

infection, and clinical manifestations between cattle, sheep, and goats. Thus, a species-specific approach is preferable (Kostoulas et al., 2006). Variable strains of MAP stimulate variable levels of antibody response in goats and cattle. The major strain types, S- and Ctype, are not host specific (de Juan et al., 2005; Sevilla et al., 2007). In a recent study in northern Greece, significant genetic diversity of MAP isolates was found in small ruminants (Dimareli-Malli et al., 2013). In terms of clinical disease, goats appeared more susceptible to MAP infection, whether infected with S- or C-type, than sheep, with cattle being the most resistant (Stewart et al., 2007). Other papers that applied the same ELISA at the recommended cutoffs in dairy cattle and goats found agreement between the proportion positive in milk ELISA with that in fecal culture, whereas the proportions positive in serum ELISA and fecal culture were not in agreement (Hendrick et al., 2005; Salgado et al., 2007).

When we lowered the recommended cutoff, we improved Se without serious loss of Sp (Table 2), which is in accordance with previous findings in goats (Kostoulas et al., 2006). The suggested optimum cutoffs that simultaneously maximize Se and Sp correspond to an informed decision that assigns equal weights to the costs of false-positive and false-negative test outcomes and implies that the prevalence in the target population is about $50 \%$. This approach may not always fully exploit the information provided by the diagnostic test in the context of a particular diagnostic objective, 
but facilitates comparison of different diagnostic tests. Cutoff selection is an informed procedure that takes into account the epidemiological situation in the target population and the relative consequences of falsenegative and false-positive test results that are defined on the grounds of a specific decision-making situation (Greiner et al., 2000) and may not necessarily be set to equal. Kostoulas et al. (2006) provided different cutoffs for the serum ELISA in sheep and goats for variable prevalence schemes and ratios of relative costs. However, our approach gives lactation-specific distributions of healthy and infected dairy goats that permit continuous interpretation of test results. The continuous interpretation eliminates the loss of information that occurs under dichotomization of continuous test results. Dichotomization of continuous results leads to a loss of valuable information because the information conveyed in the test result is reduced to considering all positive results equal. Hence, potential associations between the continuous test result and risk factors or productivity indices is attenuated or lost. A diagnostic interpretation approach that utilizes the actual continuous responses has recently been proposed (Toft et al., 2005) and utilized in the identification of the different stages of MAP infection. Decision making, such as culling or no culling, can be based on this continuous interpretation in connection with productive and reproductive indices of dairy cattle (Toft et al., 2005; Nielsen et al., 2007).

The sensitivity analysis suggested that the posterior distributions were robust under alternative prior information. Specification of more informative priors gave results similar to the primary analyses that included vague prior information. Alternative co-variance structures (compound symmetry, first-order autoregressive) were not considered because we expected an inadequate fit to our data. A correlation pattern in dairy cattle has shown that the antibodies in milk are increased at the beginning and the end of lactation (Nielsen et al., 2002a). We observed a similar trend in a preliminary analysis that was based on the calculation of the correlation of concordance (Aggelidou et al., 2012). Thus, we chose the unstructured co-variance matrix that comes at a cost in the number of parameters to be estimated but allows for estimations that are uniquely based on our data, which was preferable given the absence of relevant studies in goats. Specifying the appropriate model for within-subject correlation is essential for drawing accurate and powerful conclusions. The simplest form should be chosen but not at the expense of capturing the correct correlation pattern (Guerin and Stroup, 2000).

We also considered 2 different variations of the same model, one assuming a different prevalence of MAP infection for each lactation stage (primary analysis) and one setting MAP prevalence as constant across all lactation stages. Prevalence, AUC, Se, and Sp estimates under the alternative model specifications were similar (Table 2), indicating that, because of the low progression of MAP infection, the prevalence of MAP can be considered relatively constant during the 14-mo follow-up period. In endemic situations, MAP infection can be expected to develop slowly over time (Chiodini et al., 1984).

\section{CONCLUSIONS}

The milk ELISA can be as accurate as serum ELISA across all lactation stages and especially in late lactation. Also, because the prevalence of MAP is constant, there is no need for lactation-stage specific selection to detect the disease. The milk ELISA is preferred over the serum ELISA because milk sampling is a noninvasive, rapid, easy to apply, and low-cost procedure. Milk ELISA could serve as the diagnostic tool of choice during the implementation of MAP control programs that require frequent testing.

\section{ACKNOWLEDGMENTS}

This research has been co-financed by the European Union (European Social Fund - ESF) and Greek national funds through the Operational Program "Education and Lifelong Learning" of the National Strategic Reference Framework (NSRF) Research Funding Program: Heracleitus II. Investing in knowledge society through the European Social Fund.

\section{REFERENCES}

Aggelidou, E., P. Kostoulas, and L. Leontides. 2012. Concordance between Mycobacterium avium ssp. paratuberculosis ELISA results in paired sera and milk of dairy goats. Page 357 in Proc. 11th Int. Colloq. Paratuberculosis, Sydney, Australia. The University of Sydney, Sydney, Australia.

Bakker, D., P. T. Willemsen, and F. G. van Zijderveld. 2000. Paratuberculosis recognized as a problem at last: A review. Vet Q. $22: 200-204$.

Best, N., M. Cowles, and S. Vines. 1995. CODA: Convergence Diagnosis and Output Analysis Software for Gibbs Sampling Output. Version 0.3. MRC Biostatistics Unit, Cambridge, UK.

Branscum, A. J., I. A. Gardner, and W. O. Johnson. 2005. Estimation of diagnostic-test sensitivity and specificity through Bayesian modeling. Prev. Vet Med. 68:145-163. http://dx.doi.org/10.1016/j. prevetmed.2004.12.005.

Chiodini, R. J., H. J. van Kruiningen, and R. S. Merkal. 1984. Ruminant paratuberculosis (Johne's disease): The current status and future prospects. Cornell Vet 74:218-262.

Choi, Y.-K., W. O. Johnson, M. T. Collins, and I. A. Gardner. 2006. Bayesian inferences for receiver operating characteristic curves in the absence of a gold standard. J. Agric. Biol. Environ. Stat. 11:210-229. http://dx.doi.org/10.1198/108571106X110883. 
Clarke, C. J. 1997. The pathology and pathogenesis of paratuberculosis in ruminants and other species. J. Comp. Pathol. 116:217-261.

Coussens, P. M. 2001. Mycobacterium paratuberculosis and the bovine immune system. Anim. Health Res. Rev. 2:141-161.

de Juan, L., A. Mateos, L. Domínguez, J. M. Sharp, and K. Stevenson. 2005. Genetic diversity of Mycobacterium avium subspecies paratuberculosis isolates from goats detected by pulsed-field gel electrophoresis. Vet Microbiol. 106:249-257. http://dx.doi. org/10.1016/j.vetmic.2004.12.013.

Dimareli-Malli, Z., K. Mazaraki, K. Stevenson, P. Tsakos, A. Zdragas, V. Giantzi, E. Petridou, I. Heron, and G. Vafeas. 2013. Culture phenotypes and molecular characterization of Mycobacterium avium ssp. paratuberculosis isolates from small ruminants. Res. Vet Sci. 95:49-53. http://dx.doi.org/10.1016/j.rvsc.2013.03.010.

Florou, M., L. Leontides, P. Kostoulas, C. Billinis, and M. Sofia. 2009. Strain-specific sensitivity estimates of Mycobacterium avium ssp. paratuberculosis culture in Greek sheep and goats. Zoonoses Public Health 56:49-52. http://dx.doi.org/10.1111/j.18632378.2008.01179.x.

Fluss, R., D. Faraggi, and B. Reiser. 2005. Estimation of the Youden index and its associated cutoff point. Biom. J. 47:458-472.

Gardner, I. A., S. S. Nielsen, R. J. Whittington, M. T. Collins, D Bakker, B. Harris, S. Sreevatsan, J. E. Lombard, R. Sweeney, D. R. Smith, J. Gavalchin, and S. Eda. 2011. Consensus-based reporting standards for diagnostic test accuracy studies for paratuberculosis in ruminants. Prev. Vet Med. 101:18-34.http://dx.doi. org/10.1016/j.prevetmed.2011.04.002.

Greiner, M., D. Pfeiffer, and R. D. Smith. 2000. Principles and practical application of the receiver-operating characteristic analysis for diagnostic tests. Prev. Vet Med. 45:23-41.

Guerin, L., and W. W. Stroup. 2000. A simulation study to evaluate PROC MIXED analysis of repeated measures data. Page 170-203 in Proc. Conf. Appl. Stat. Agric., Kansas State University, Manhattan, KS. http://www.kstate.edu/stats/agstat. conference/2000_table_of_contents.htm.

Hendrick, S. H., T. E. Duffield, D. E. Kelton, K. E. Leslie, K. D. Lissemore, and M. Archambault. 2005. Evaluation of enzyme-linked immunosorbent assays performed on milk and serum samples for detection of paratuberculosis in lactating dairy cows. J. Am. Vet Med. Assoc. 226:424-428.

Hernández-Castellano, L. E., A. Torres, A. Alavoine, M. D. Ruiz-Díaz, A. Argüello, J. Capote, and N. Castro. 2011. Effect of milking frequency on milk immunoglobulin concentration (IgG, IgM and IgA) and chitotriosidase activity in Majorera goats. Small Rumin. Res. 98:70-72. http://dx.doi.org/10.1016/j.smallrumres.2011.03.021.

Jafarzadeh, S. R., W. O. Johnson, J. M. Utts, and I. A. Gardner. 2010. Bayesian estimation of the receiver operating characteristic curve for a diagnostic test with a limit of detection in the absence of a gold standard. Stat. Med. 29:2090-2106. http://dx.doi. org/10.1002/sim.3975.

Kennedy, D. J., and G. Benedictus. 2001. Control of Mycobacterium avium ssp. paratuberculosis infection in agricultural species. Rev. Off. Int. Epizoot. 20:151-179.

Kostoulas, P., W. J. Browne, S. S. Nielsen, and L. Leontides. 2013. Bayesian mixture models for partially verified data: Age- and stage-specific discriminatory power of an antibody ELISA for paratuberculosis. Prev. Vet Med. http://dx.doi.org/10.1016/j. prevetmed.2013.05.006.

Kostoulas, P., L. Leontides, C. Enøe, C. Billinis, M. Florou, and M. Sofia. 2006. Bayesian estimation of sensitivity and specificity of serum ELISA and faecal culture for diagnosis of paratuberculosis in Greek dairy sheep and goats. Prev. Vet Med. 76:56-73. http:// dx.doi.org/10.1016/j.prevetmed.2006.04.006.

Kumar, S., S. V. Singh, I. Sevilla, A. V. Singh, R. J. Whittington, R. A. Juste, G. Sharma, P. K. Singh, and J. S. Sohal. 2008. Lactoprevalence, genotyping of Mycobacterium avium subspecies paratuberculosis and evaluation of three diagnostic tests in milk of naturally infected goat herds. Small Rumin. Res. 74:37-44. http:// dx.doi.org/10.1016/j.smallrumres.2007.03.005.

Lombard, J. E., T. M. Byrem, B. A. Wagner, and B. J. McCluskey. 2006. Comparison of milk and serum enzyme-linked immu- nosorbent assays for diagnosis of Mycobacterium avium subspecies paratuberculosis infection in dairy cattle. J. Vet Diagn. Invest. 18:448-458.

Lybeck, K. R., A. K. Storset, B. Djønne, M. Valheim, and I. Olsen. 2011. Faecal shedding detected earlier than immune responses in goats naturally infected with Mycobacterium avium ssp. paratuberculosis. Res. Vet Sci. 91:32-39. http://dx.doi.org/10.1016/j. rvsc. 2010.08.012

Moreno-Indias, I., D. Sánchez-Macías, N. Castro, A. MoralesdelaNuez, L. E. Hernández-Castellano, J. Capote, and A. Argüello. 2012. Chemical composition and immune status of dairy goat colostrum fractions during the first $10 \mathrm{~h}$ after partum. Small Rumin. Res. 103:220-224. http://dx.doi.org/10.1016/j. smallrumres.2011.09.015.

Nielsen, S. S. 2002. Variance components of an enzyme-linked immunosorbent assay for detection of IgG antibodies in milk samples to Mycobacterium avium subspecies paratuberculosis in dairy cattle. J. Vet Med. B Infect. Dis. Vet Public Health 49:384-387.

Nielsen, S. S., Y. T. Gröhn, and C. Enevoldsen. 2002a. Variation of the milk antibody response to paratuberculosis in naturally infected dairy cows. J. Dairy Sci. 85:2795-2802. http://dx.doi. org/10.3168/jds.S0022-0302(02)74366-X.

Nielsen, S. S., C. Grønbæk, J. F. Agger, and H. Houe. 2002b. Maximum-likelihood estimation of sensitivity and specificity of ELISAs and faecal culture for diagnosis of paratuberculosis. Prev. Vet Med. 53:191-204.

Nielsen, S. S., N. Toft, E. Jørgensen, and B. M. Bibby. 2007. Bayesian mixture models for within-herd prevalence estimates of bovine paratuberculosis based on a continuous ELISA response. Prev. Vet Med. 81:290-305. http://dx.doi.org/10.1016/j.prevetmed.2007.05.014.

Ntzoufras, I. 2009. Bayesian Modeling Using WinBUGS. J. Wiley and Sons Inc., Hoboken, NJ.

Pritchett, L. C., C. C. Gay, T. E. Besser, and D. D. Hancock. 1991. Management and production factors influencing immunoglobulin G1 concentration in colostrum from Holstein cows. J. Dairy Sci. 74:2336-2341.

Raftery, A. L., and S. Lewis. 1992. Comment: One long run with diagnostics: Implementation strategies for Markov chain Monte Carlo. Stat. Sci. 7:493-497.

Salgado, M., J. Kruze, and M. T. Collins. 2007. Diagnosis of paratuberculosis by faecal culture and ELISA on milk and serum samples in two types of Chilean dairy goat herds. J. Vet Diagn. Invest. 19:99-102. http://dx.doi.org/10.1177/104063870701900117.

Salgado, M., E. J. B. Manning, and M. T. Collins. 2005. Performance of a Johne's disease enzyme- linked immunosorbent assay adapted for milk samples goats. J. Vet Diagn. Invest. 17:350-354.

Sevilla, I., J. M. Garrido, M. Geijo, and R. A. Juste. 2007. Pulsed-field gel electrophoresis profile homogeneity of Mycobacterium avium ssp. paratuberculosis isolates from cattle and heterogeneity of those from sheep and goats. BMC Microbiol. 12:7-18.

Spiegelhalter, D., A. Thomas, N. Best, and W. Gilks. 1996. BUGS Bayesian Inference using Gibbs Sampling. Version 0.50. MRC Biostatistics Unit, Cambridge, UK.

Stabel, J. R. 2000. Transitions in immune responses to Mycobacterium paratuberculosis. Vet Microbiol. 77:465-473.

Stehman, S. M. 1996. Paratuberculosis in small ruminants, deer, and South American camelids. Vet Clin. North Am. Food Anim. Pract. 12:441-455.

Stewart, D. J., J. A. Vaughan, P. L. Stiles, P. J. Noske, M. L. V. Tizard, S. J. Prowse, W. P. Michalski, K. L. Butler, and S. L. Jones. 2007. A long-term bacteriological and immunological study in Holstein-Friesian cattle experimentally infected with Mycobacterium avium ssp. paratuberculosis and necropsy culture results for Holstein-Friesian cattle, Merino sheep and Angora goats. Vet Microbiol. 122:83-96. http://dx.doi.org/10.1016/j.vetmic.2006.12.030.

Storset, A. K., H. J. Hasvold, M. Valheim, H. Brun-Hansen, G. Berntsen, S. K. Whist, B. Djønne, C. M. Press, G. Holstad, and H. J. Larsen. 2001. Subclinical paratuberculosis in goats following experimental infection. An immunological and microbiological study. Vet Immunol. Immunopathol. 80:271-287. 
Toft, N., S. S. Nielsen, and E. Jørgensen. 2005. Continuous-data diagnostic tests for paratuberculosis as a multistage disease. J Dairy Sci. 88:3923-3931. http://dx.doi.org/10.3168/jds.S00220302(05)73078-2.

Wang, C., B. W. Turnbull, Y. T. Gröhn, and S. S. Nielsen. 2007. Nonparametric estimation of ROC curves based on Bayesian models when the true disease state is unknown. J. Agric. Biol. Environ. Stat. 12:128-146.
Wang, C., B. W. Turnbull, S. S. Nielsen, and Y. T. Gröhn. 2011 Bayesian analysis of longitudinal Johne's disease diagnostic data without a gold standard test. J. Dairy Sci. 94:2320-2328. http:// dx.doi.org/10.3168/jds.2010-3675.

Zygogiannis, D., and N. Katsaounis. 1992. Goats Breeding. 1st ed. D. Zygogiannis and N. Katsaounis, Thessaloniki, Greece. (in Greek). 